\title{
Model error estimation based on asymptotic homogenization for periodic heterogeneous structures
}

\author{
Mouad Fergoug*, $\dagger \ddagger$, Nicolas Feld ${ }^{\ddagger}$, Samuel Forest ${ }^{\dagger}$, Basile Marchand ${ }^{\dagger}$ and Augustin \\ Parret-Fréaud ${ }^{\ddagger}$ \\ $\dagger$ MINES ParisTech, PSL University \\ MAT - Centre des matériaux \\ CNRS UMR 7633, BP 8791003 Evry, France \\ $\ddagger$ Safran Tech \\ Etablissement Paris Saclay \\ Rue des Jeunes Bois-Châteaufort, 78114 Magny-les-Hameaux, France
}

\begin{abstract}
Traditional computational analyses of periodic structures involve the use of periodic homogenization [1], [2] to achieve computational feasibility and efficiency instead of conducting a full field heterogeneous analysis. A modeling error [3] is therefore induced due to the difference between the homogenized and heterogeneous models. We propose an estimate of this modeling error associated with homogenization of composite structures. The proposed estimate is constructed based on the difference between the solution of the classical first-order homogenization problem with boundary layer effect correction [4] and the solutions of higher-order problems [5] (up to the third-order) resulting from asymptotic expansion method. The technique enables the detection of regions where the computational model needs to be modeled on the microscopic scale instead of the macroscopic homogeneous model. The modeling error estimate is implemented in the finite element code Zset [6], its efficiency and accuracy were verified by means of numerical examples.
\end{abstract}

\section{REFERENCES}

[1] Sanchez Palencia, E. Nonhomogeneous Media and Vibration Theory. Lecture Note in Physics, $127(1980)$.

[2] Boutin, C. Microstructural effects in elastic composites. International Journal of Solids and Structures (1996) 33:1023 - 1051.

[3] Zohdi, T I and Oden, J T and Gregory J R. Hierarchical modeling of heterogeneous bodies.Computer Methods in Applied Mechanics and Engineering (1996) 138:273 - 298.

[4] Dumontet, H. Boundary Layers Stresses in Elastic Composites. Studies in Applied Mechanics (1985) 12:215 - 232.

[5] Fish, J and Nayak, $\mathrm{P}$ and Holmes, M H. Microscale reduction error indicators and estimators for a periodic heterogeneous medium. Computational Mechanics (1994) 14:323-338.

[6] Z-set, Non-linear material \& structure analysis suite, www.zset-software.com. 